\title{
An Edge Detection Algorithm of Anti-Harmonic Image Based on Tensor Form
}

\author{
Li Xiang* $^{*}$
}

Ling Nan Normal University, Zhanjiang, 524028, China

\begin{abstract}
Edge detection of image is used to improve the visual recognition ability of the pattern by identifying points with obvious variation of brightness in a digital image. In the case of uneven conversion of color information of antiharmonic image pixel, edge detection becomes difficult. Traditional edge detection algorithm of anti-harmonic image adopts tensor model, as due to relatively simple structure of the tensor vector and operation, the performance of edge detection is poor. Therefore, An edge detection algorithm of anti-harmonic image based on colored tensor form is proposed. It starts from building the tensor model of colored and anti-harmonic image, accurately measuring the relationship between pixels by means of rich computing of tensor, finding the maximum and minimum values of the first derivative of the image to detect the boundary in the space of tensor form, and finally improve the algorithm based on the color tensor morphological operators of referenced total order. Simulation results show that the anti-harmonic mean of the proposed algorithm is higher than the conventional algorithms because it not only takes into account the correlation between the color components, but also considers the conversion feature of color information, which has superior performance of detection.
\end{abstract}

Keywords: Anti-harmonic, edge detection, image, tensor form.

\section{INTRODUCTION}

With the development of computer image processing technology, digital image processing technology of computer graphics school is gradually applied in the fields of remote sensing image analysis, long-range target detection and artificial intelligence, etc. Edge detection and contour decomposition of image are important methods of extracting contour information feature of colored image through image segmentation; it involves zoning physical information characteristics of image such as grey level, color and texture, looking for the similarity and consistency, and realizing analysis and processing of the detail of the image. The purpose of edge detection is to identify points which have apparent brightness that changes in digital image, rejects the irrelevant information, and keeps important structural attribute of the image. Colored image contains more rich information, but lacks of more clear order relation, whose edge detection appears relatively difficult. It can clearly be seen that color information of pixels of anti-harmonic image changes unevenly, and edge detection has always been the difficulties of colored image fusion and analysis, so the relevant algorithms are brought to the attention [1].

Among traditional methods of edge detection, edge detection algorithms for colored anti-harmonic images can be mainly divided into two classes: one category is based on the search and the other is based on zero crossing, including edge algorithms, such as the edge detection algorithm based

\footnotetext{
*Address correspondence to this author at the Ling Nan Normal University, Zhanjiang, 524028, China; Tel: 18986139113:

E-mail: Hunter2011@foxmail.com
}

on grey histogram [1], the edge detection algorithm based on gradient operator [2], the edge detection algorithm based on signal-to-noise ratio of image [3, 4], etc. In which, reference [5] has proposed an edge extraction method based on greylevel histogram, reducing the amount of computation and producing good edge effect, while the algorithm has nonlinear differential effect, which brings about poor performance on the recognition of edge detection. Literature [6] has proposed an edge detection algorithm based on offset field correction of section image, effectively and accurately extracting the edge information of image section, performing quantitative analysis of the correlation of image motion and the image parameters, while the algorithm may extract excess contour line which generates the leak and disorder of edge information detection. Reference [7] uses tensor model algorithm to detect the edge of anti-harmonic image, where brightness, contrast and structural similarity of image are the baseline characteristics of scene structure, realizing the edge detection, while the performance of edge detection is poor due to simple structure and operation of tensor vector [8-11].

Aiming at the above problems, in order to improve the performance of edge detection of colored anti-harmonic images and improve the ability of identification and decomposition of images, an algorithm of anti-harmonic image edge detection based on colored tensor form has been proposed. It involves constructing the tensor model of colored antiharmonic image, designing anti-harmonic and average morphology operator, improving the algorithm based on the reference total order colored tensor morphological operator, and realizing the improvement of edge detection algorithm of anti-harmonic image. The simulation experiment has done 
performance testing and verification, which reveals effective conclusions.

\section{TENSOR MODEL AND FEATURE EXTRACTION OF EDGE DETECTION OF ANTI-HARMONIC IM- AGE}

\subsection{Design of Tensor Model for Edge Detection of Anti- harmonic Image}

Edge detection of image can significantly reduce the amount of computation. Although, there are many methods for edge detection, yet we have proposed a modified edge detection method based on tensor model. Through related definitions, tensor is a multi-linear function which defies on Cartesian product in some vector space and some dual space, finding the boundary by looking for zero crossing of second derivative of the image, receiving a tensor space, depicting the color information of each pixel through second-order two-dimensional tensor; the tensor space of the image can be expressed as a $2 \times 2$ matrix:

$\mathbf{T}=\left(\begin{array}{ll}T_{11} & T_{12} \\ T_{21} & T_{22}\end{array}\right)$

Thus, the problem of edge detection of anti-harmonic image has been converted to the problem of solving characteristic value of tensor model space above, that is, $T-(\lambda I) e=0$ characteristic values $\lambda_{1}$ and $\lambda_{2}\left(\lambda_{1} \geq \lambda_{2}\right)$ the direction of main edge characteristic of anti-harmonic image is defined as $e_{1}$.

For many second-order two-dimensional symmetric positive definite tensor, the pixel information of anti-harmonic image in the case of colored space can be described as:

$h=\theta / \pi, \quad s=1-\frac{\lambda_{2}}{\lambda_{1}}, \quad v=\lambda_{1}+\lambda_{2}$

where, $\theta$ is the main direction angle of ellipse, $\lambda_{1}, \lambda_{2}$ are the lengths of long and short half axes, respectively, building HSV colored space, considering the color image $F$ in domain $E: E \rightarrow R^{3}$, setting $\mathbf{T}: E \rightarrow \operatorname{PDS}(2)$, thus tensor representation of color information of anti-harmonic image is obtained, which is shown in Fig. (1).

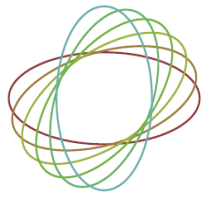

(a) $\mathrm{H}$

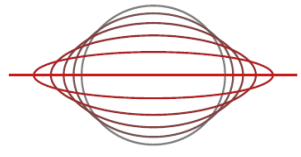

(b) $\mathrm{S}$

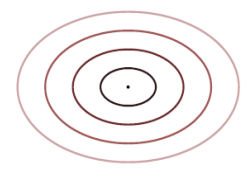

(c) $\mathrm{V}$
Fig. (1). Tensor information of color information of anti-harmonic image.

\subsection{Feature Extraction of Anti-Harmonic Color Image}

According to Fig. (1), many second order twodimensional symmetric positive definite tensor can only determine an ellipse, above on the tensor space; by extracting the feature of colored image tensors that are shot in the same environment at the same time, the initial pixel value of the anti-harmonic image is obtained; and by computing hierarchy window of the reference images, the image to be matched is achieved, the position error of image is:

$$
\begin{aligned}
& I_{l}\left(n_{1}, n_{2}\right)=\frac{1}{4} \sum_{i=0}^{1} \sum_{i=0}^{1} I_{l-1}\left(2 n_{1}+i_{1}, 2 n_{2}+i_{2}\right) \\
& J_{l}\left(n_{1}, n_{2}\right)=\frac{1}{4} \sum_{i=0}^{1} \sum_{i 2=0}^{1} J_{l-1}\left(2 n_{1}+i_{1}, 2 n_{2}+i_{2}\right)
\end{aligned}
$$

A $7 \times 7$ pixel matching window (selecting image area in the center of the reference point), consists of an antiharmonic interference vector, for building the phase correlation function of image matching problem, whose essence is the two-dimensional discrete Fourier inversion of mutual phase spectral function; the inversion characteristic function is:

$r\left(n 1, n_{2}\right)=\frac{1}{N_{1} N_{2}} \sum_{k_{1} k 1} R\left(k_{1}, k_{2}\right) e^{j \frac{2 \pi k 1 n 1}{N 2}} e^{j \frac{2 \pi k 2 n 2}{N 2}}$

The above equation defines mutual phase correlation function, that is if two images are completely dissimilar, the two-dimensional characteristics of mutual phase spectral function $R\left(k_{1}, k_{2}\right)$ are as follows:

$R\left(k_{1}, k_{2}\right)=\frac{F\left(k_{1}, k_{2}\right) \overline{G\left(k_{1}, k_{2}\right)}}{\left|F\left(k_{1}, k_{2}\right) \overline{G\left(k_{1}, k_{2}\right)}\right|}=e^{j \theta\left(k_{1}, k_{2}\right)}$

Set the gray image $f$, for any $\operatorname{pixel}(x, y)$, assuming the local neighborhood determined by flat structure elements is $B(x, y)$, using Atanassov to expand the fuzzy membership function, the intuitionistic fuzzy set $u=\left\{u_{i k}\right\}$ of tensor form is given, in the fuzzy set scale space. By using the twodimensional planar discrete zero padding method, the image is enhanced followed by extending the matrix for the extracting edge of the contour feature, setting $A=\left\{a_{i}\right\}_{i=1}^{N}$ as a collection of $N$ scalar, and $P$ order anti-harmonic average (CHM) is as follows:

$\kappa^{P}(A)=\left(\sum_{i=1}^{N} a_{i}^{P}\right)^{-1 / 2}\left(\sum_{i=1}^{N} a_{i}^{P+1}\right)\left(\sum_{i=1}^{N} a_{i}^{P}\right)^{-1 / 2}$

According to the colored information of pixels, the tensor spectrum can be achieved reasonably, which satisfies the following properties:

$$
\begin{gathered}
s \leq t \Rightarrow \kappa^{s}(A) \leq \kappa^{t}(A) \\
\lim _{P \rightarrow+\infty} \kappa^{P}(A)=\max _{i} a_{i}
\end{gathered}
$$

$\lim _{P \rightarrow-\infty} \kappa^{P}(A)=\min _{i} a_{i}$

Therefore, constructing colored morphological operator by means of the rich operation and properties of tensor, will help detect the boundary by looking for the maximum and minimum values of the first derivative, and extract the features of colored anti-harmonic image, which provides characteristic information foundation for the edge detection of anti-harmonic image. 
3. COLOR TENSOR MORPHOLOGICAL OPERATORS BASED ON REFERENCED TOTAL ORDER AND IMPROVEMENT OF EDGE DETECTION

\subsection{Color Tensor Morphological Operators Based on the Referenced Total Order}

In the foundation of the tensor space building and above feature extraction, designing anti-harmonic and average morphology operator, and finding the maximum and minimum value of the first order derivative image in tensor form space to detect edges, are sought. Setting $\mathbf{A}=\left\{\mathbf{A}_{i}\right\}_{i=1}^{N}$ as $N$ symmetric positive definite tensor, two pixels respectively satisfy:

$$
\begin{aligned}
& \lim _{P \rightarrow+\infty} \kappa_{B}^{P}(f)(x, y)=\max _{(s, t) \in B(x, y)} f(s, t)=\delta_{B}(f)(x, y) \\
& \lim _{P \rightarrow-\infty} \kappa_{B}^{P}(f)(x, y)=\min _{(s, t) \in B(x, y)} f(s, t)=\varepsilon_{B}(f)(x, y)
\end{aligned}
$$

Among them, $\delta$ and $\varepsilon$ represent a scalar and antiharmonic operator respectively. Select one or a set of reference value through certain guidelines based on the sorting method of reference value, and the tensor total of antiharmonic average is obtained:

$\delta_{B}^{T}(F)(x, y)=\lim _{P \rightarrow+\infty} \kappa_{B}^{P}(\mathbf{T})(x, y)=\lim _{P \rightarrow+\infty} \kappa^{P}\left(\mathbf{A}_{(x, y)}\right)$

Using the datum feature extraction method, extract brightness, contrast and structural similarity from antiharmonic image as the baseline characteristics of scene structure. The morphological operator $\kappa_{B}^{P}(\mathbf{T})$ based on antiharmonic average is a kind of hierarchical (rank) morphology filter, by setting $\mathbf{A}=\left\{\mathbf{A}_{i}\right\}_{i=1}^{N}$ as $N$ symmetric positive definite tensor, reference total order colored tensor morphological operator is defined as follows:

$\delta_{B}^{T}(F)(x, y)=\vee_{(\mathbf{S}, \mathbf{I})} \mathbf{A}_{(x, y)}$

$\varepsilon_{B}^{T}(F)(x, y)=\wedge_{(\mathbf{S}, \mathbf{I})} \mathbf{A}_{(x, y)}$

where, $B$ is the structure elements of the morphological operator, for the choice of the reference tensor, using the colored tensor morphological operator of reference sequence to calculate, thereby improving the matching performance of edge detection.

\subsection{Achievement of Edge Detection Algorithm}

Based on the above model of colored tensor morphology operator, this paper puts forward a kind of anti-harmonic image edge detection algorithm based on colored tensor form, realizing the improvement of the anti-harmonic image edge detection algorithm; and the total order relation of the tensor $(\mathbf{S}, \mathbf{I})$ of the anti-harmonic image can be obtained through analysis:

$$
\begin{aligned}
& \mathbf{A}_{i} \leq_{(\mathbf{S}, \mathbf{I})} \mathbf{A}_{j} \Leftrightarrow h_{(\mathbf{S}, \mathbf{I})}\left(\mathbf{A}_{i}\right) \leq h_{(\mathbf{S}, \mathbf{I})}\left(\mathbf{A}_{j}\right) \\
& h_{(\mathbf{S}, \mathbf{I})}(\mathbf{A})=K(\mathbf{S}, \mathbf{A})-K(\mathbf{I}, \mathbf{A})
\end{aligned}
$$

Reference total order colored tensor form mapping $h_{(\mathbf{S}, \mathbf{I})}(\mathbf{A})$ reflects the distance relationship between tensor $\mathbf{A}$ and the anti-harmonic image edge reference tensor $(\mathbf{S}, \mathbf{I})$. In which, mapping $K: \operatorname{POS}(n) \times \operatorname{POS}(n) \rightarrow R^{+} \cup\{0\}$, considering the color image $F: E \rightarrow R^{3}(x, y) \in E$ in the discourse region $E$, making the edge character $\mathbf{A}_{(x, y)}=\{\mathbf{T}(s, t) \mid(s, t) \in B(x, y)\}$, visibly, the anti-harmonic average is qualitative, which can correspond to the threedimensional vector one by one in HSV colored space, and brightness of the corresponding colored pixel is reduced, which is in line with the gray situation, thus realizing the improvement of edge detection of the anti-harmonic image.

\section{SIMULATION EXPERIMENT AND RESULT ANALYSIS}

To test the performance of the algorithm in the realization of the edge detection of the anti-harmonic image, the simulation experiment has been done. Simulation hardware environment is: IntelCore $2.4 \mathrm{GHz} \mathrm{CPU}$ and a computer with memory of $1 \mathrm{~GB}$, and the simulation software is COMSOL Multiphysics finite element analysis software. The collector of colored anti-harmonic image is a panchromatic sensor with $1 \mathrm{~m}$ resolution and a multispectral sensor image acquisition model with four band $4 \mathrm{~m}$ resolution, in setting of image parameters, assuming two pixels under tensor form colored space are $a_{1}=(0.4,0.9,0.9), a_{2}=(0.6,0.7,0.6)$, respectively. Firstly image feature extraction pretreatment is done, selected optimal $\beta$ parameters are: 3.2, 1.2 and 3.5. The second order tensor designed is:

$$
\begin{aligned}
& \mathbf{A}_{1}=\left(\begin{array}{ll}
0.1521 & 0.2164 \\
0.2164 & 0.7479
\end{array}\right), \\
& \mathbf{A}_{2}=\left(\begin{array}{cc}
0.1693 & -0.0949 \\
-0.0949 & 0.4307
\end{array}\right)
\end{aligned}
$$

Taking $\mathrm{P}=-5 \sim 15$, calculate the anti-harmonic average results of two tensors, and simulation results of the edge detection of anti-harmonic colored image based on tensor form designed by the article are shown in Fig. (2).

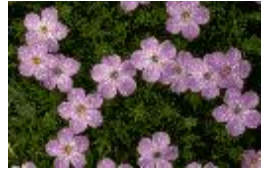

(a) the original picture

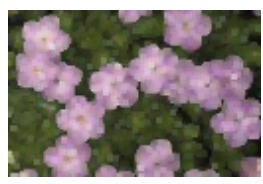

(d) $\mathrm{P}=5$

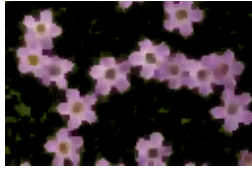

(b) $\mathrm{P}=-5$

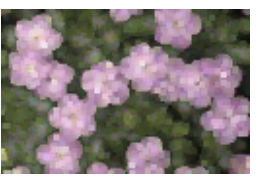

(e) $\mathrm{P}=10$

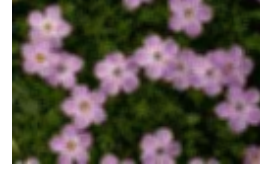

(c) $\mathrm{P}=0$

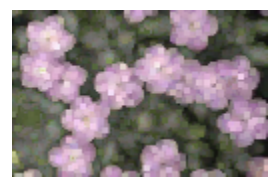

(f) $\mathrm{P}=15$
Fig. (2). Process simulation of edge detection of anti-harmonic color image based on tensor form.

Analysis of diagram 2 shows, using the algorithm in this paper, with the unceasing change of order, color of the antiharmonic image gradually changes from dark to bright after 


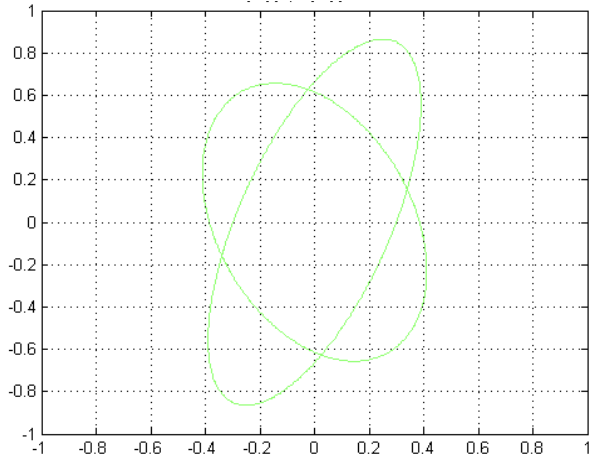

(a) traditional algorithm

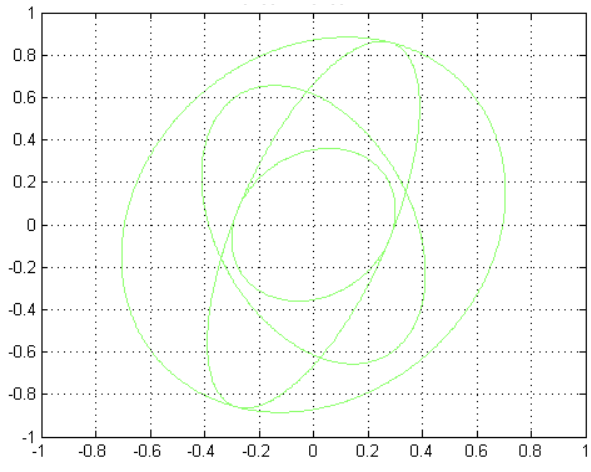

(b) algorithm in this paper

Fig. (3). Anti-harmonic average results of edge detection tensor.

edge detection, effectively identifies points with clear brightness changes in digital image, and eliminates information which can be seen as irrelevant, retaining the important structural attributes of the image, realizing accurate detection of the image edge. Algorithm in this paper measures the relationship between pixels more accurately by rich operation of tensor. To contrast performance advantages of algorithms, using this algorithm and traditional algorithm, take the antiharmonic mean as test indicator to compare detection performance, and the results are shown in Fig. (3). Analysis of diagram 3 shows that algorithm in this article has a higher anti-harmonic mean, and depicting the color information by tensor model can not only consider the correlation between color components, but also consider the transform characteristics of color information, which has a higher accuracy of edge detection.

\section{CONCLUSION}

Edge detection can effectively identifies points with clear brightness changes in digital image, and eliminates information which can be seen as irrelevant, keeping the important structural attributes of the image. Colored images contain more abundant information, due to the color information lack of specific total order relationship, whose edge detection is relatively difficult. In order to improve the performance of edge detection of anti-harmonic colored image, an anti-harmonic image edge detection algorithm based on colored tensor form has been proposed in this paper, and the experimental analysis shows that algorithm can effectively improve the performance of image edge detection, which has a higher anti-harmonic average results. Depicting the color information by tensor model can not only consider the correlation between color components, but also consider the transform features of colored information, which shows superior performance.

\section{CONFLICT OF INTEREST}

The author confirms that this article content has no conflict of interest.

\section{ACKNOWLEDGEMENTS}

This work is supported by the Key Project of Science and Technology research project of Henan province: (Research of dynamic vehicle positioning system based on digital video image, No. 132102210423).

\section{REFERENCES}

[1] L. Min, and L. Jun, "Based on the characteristics of human vision system image quality evaluation algorithm," Bulletin of Science and Technology, vol. 29, no. 2, pp. 1-6, 2013.

[2] S. Han, W.Tao, and X. Wu, "Texture segmentation using independent-scale component-wise Riemannian-covariance Gaussian mixture model in KL measure based multi-scale nonlinear structure tensor space ," Pattern Recognition, vol. 44, pp. 503-518, 2011.

[3] L.Rittner, F.C.Flores, and R.A.Lotufo, "A tensorial framework for color images,”Pattern Recognition Letters, vol. 31, pp. 277-296, 2010.

[4] B.Burgeth, L.Pizarro, and M.Breuss, "Adaptive continuous-scale morphology for matrix fields," International Journal of Computer Vision, vol. 92, pp. 146-161, 2011

[5] Y.Jing-yu, Z. Yong-sheng, and Z. Xiao-liang, "Using dark channel prior to quickly remove haze from a single image," Geomatics and Information Science of Wuhan University, vol. 35, no. 11, pp. 1-8, 2011.

[6] L. Liang, W. Tong-qing, and Y. Bo,'Research of image association rules mining," Application Research of Computers, vol. 26, no. 6, pp. 1-5, 2009.

[7] L. Yan-ling, H.Chun-yan, and Z. Juan, “ An adaptive algorithm for median filter of image based on grey relation,"Computer Simulation, vol. 27, no. 1, 2010.

[8] L. Hai-chi, L. Yue-yang, and S.Jun, "Filtering method for images based on adaptive neuro-fuzzy inference system," Computer Science, vol. 40, no. 7, pp. 1-8, 2013.

[9] D. Hui, "Fast face detection in color image based on wavelet transform," Bulletin of Science and Technology, vol. 28, no. 12, pp. 1012012, 2012.

[10] S. Hong-hui, W. Hong-xia, and T. Tao, "The recognition method of objects based on moment invariant and BP neural network," Microelectronics \& Computer, vol. 28, no. 3, pp. 113-118, 2011.

[11] D.S. Massey, J. Arango, G. Hugo, A. Kouaouci, A. Pellegrino, and J.E. Taylor, "An evaluation of international migration theory: The North American case," Population Devision Review, vol. 20, pp. 699-751, 1994. 